Special issue of the 3rd International Conference on Computational and Experimental Science and Engineering (ICCESEN 2016)

\title{
An Efficient Inductive Coil Link Design for Wireless Power Transfer to Visual Prostheses
}

\author{
M.E. CELIK* AND E. Aydin \\ Gazi University, Department of Electrical-Electronics Engineering, Ankara, Turkey
}

\begin{abstract}
Powering therapeutic devices plays a crucial role in the design phase of implantable electronic units. Batteries could not provide sufficient charge storage, used for the lifetime of the device, considering the space available for them. As sophisticated surgical procedures are required with the possible trauma to the patient such as material discrepancy and tissue damage, replacement is quite risky and not an option. Thus, therapeutic devices, especially visual prostheses, receive power wirelessly through inductive coil links which are connected to a battery outside the body. An inductive link is based on the main principle that there are two components such as primary and secondary units. Although primary unit includes the battery outside the body, power transmitter, and control unit, secondary unit under the skin contains amplifier, rectifier regulators, and power control unit. In this study, an inductive coil link is modelled to evaluate fundamental design specifications for visual prostheses. Inductive coil link parameters are quantitatively investigated so that maximum power transfer efficiency and miniaturization, which are critically important for electronics placed in intraocular region, are provided. Considering $20 \mathrm{~mm}$ distance, it is concluded that the separation and the size of the coil pair, loop diameter, frequency have a major role to determine the system performance.
\end{abstract}

DOI: 10.12693/APhysPolA.132.535

PACS/topics: wireless power transfer, inductive link, magnetic field, telemetry, visual prosthesis

\section{Introduction}

Biomedical implants are artificial devices that are implanted in the human body to replace damaged organs which are responsible for vital functions in the body. Such devices are permanently sealed with a biocompatible sheath and contain rechargeable batteries. Because of physical constraints, it cannot pass through tissue and skin, and because of the irreplaceable nature, batteries for these devices need to be charged wirelessly from outside the body, which is called transcutaneous power transfer. Biomedical devices implanted in the body carry unchangeable batteries. Because of the location of the devices and the risk of damage to the surrounding tissue, establishing a cable connection may not be suitable for long-term usage. Therapeutic devices implanted in the body should be therefore wirelessly charged.

Nowadays, implantable medical devices are getting more important technology because it was stated that $8 \%$ to $10 \%$ of the population in America alone and $5 \%$ to $6 \%$ of people in industrialized countries have tried an implantable medical device for regenerating functions of the organs, providing a better quality of life, or expanding mobility. Implants are used in many different region of the body for certain applications such as deep brain stimulation which is used for the treatment of movement disorders such as Parkinson's disease and chronic pain, pacemakers, defibrillators which check the heartbeat for an abnormal rhythm, neural prosthetics such as cochlear

*corresponding author; e-mail: mahmutemincelik@gazi.edu.tr implants which help patients to hear for better understanding of the speech and visual prosthesis which aims to restore visual perception for blind patients [1]. Certain parameters of the biomedical devices are briefly listed in Table I.

\section{TABLE I}

Properties of certain biomedical devices using wireless power transfer.

\begin{tabular}{c|c|c|c}
\hline \hline Implant type & Power used & Distance $[\mathrm{mm}]$ & Coil dimension \\
\hline cardio & $15 \mathrm{~mW}-20 \mathrm{~W}$ & $10-25$ & $400 \mathrm{~mm}^{2}$ \\
\hline cochlear & $15 \mathrm{~mW}$ & $10-25$ & $100 \mathrm{~mm}^{2}$ \\
\hline neural & $20 \mathrm{~mW}$ & $5-25$ & $100 \mathrm{~mm}^{2}$ \\
\hline retinal & $50 \mathrm{~mW}$ & $20-25$ & $64 \mathrm{~mm}^{2}$
\end{tabular}

In this study, it is reviewed that wireless power transfer mechanisms are used for biomedical devices and best choice for a visual prosthesis system. We investigated the mechanisms used for wireless power transfer in biomedical devices implanted in the body, especially visual prosthesis devices and investigated what should be performed on choosing the topology, load, and working frequency in order to increase the power transfer efficiency for an inductive power transfer mechanism. An inductive power transfer mechanism has been basically developed using certain design criteria for maximum power transfer under magnetostatic conditions. It is seen that the distance between coils has a critical effect on the performance of the system in addition to frequency, load, and current.

\section{Wireless power transfer concept}

Providing power transmission from one device to another without a conductive medium such as a cable is 
called wireless power transfer. Wireless power transfer can be achieved by electrical, magnetic, or electromagnetic coupling. The principle of operation of the link involves the generation of magnetic field by moving electrical charges in one of the coil. This magnetic field induces an electromotive force (EMF) when a second coil is placed close to it. The coupling factor is given by the following equation:

$$
k=\frac{M}{\sqrt{L_{1} L_{2}}},
$$

where $M$ is the mutual inductance between the coils. The quality factor $Q$ represents properties of the coil such as coil geometry and the electrical property of its material

$$
2 Q=\frac{\omega L}{R},
$$

where $\omega, R$ are respectively radial frequency and coil resistance.

There are three different methods which are widely used.

Firstly, inductive power transfer (IPT) is a method in which common inductance is used to transfer power between two inductances to another. Initially, this issue, which was addressed by Tesla, has been extensively studied and provides wireless transfer of up to $40 \mathrm{~mm}$ in industrial, robotic, and biomedical applications. When a current that changes with time is applied to the first coil, a varying magnetic field occurs. This magnetic field induces a voltage at the second coil ends according to the Faraday electromagnetic induction law. In this method, which is near-field coupling, power transfer is provided by the common inductance between the two coils. Initial work in this area was based on the early 1960s, when the feasibility of power transfer from a tissue barrier was made $[2,3]$. This work was later provided with a completely wireless implanted device by integrating data transfer into the existing system [4]. Subsequent studies have been conducted by different groups from 1965 to 1990 , with respect to resonant tuning, increasing power transfer capacity, coil miniaturization, magnetic core usage, transmitter power control, wideband transfer and insensitive power transfer [5-8].

Secondly, in capacitive power transfer, the transmitter and receiver sides are planar metal plates and together form a capacitor. In this method of using near-field coupling, the distance should be less than $10 \mathrm{~mm}$. With this approach, it is not used in biomedical applications due to the large space requirement required in short intervals, not exceeding the specific absorption rate (SAR) limit, in order to obtain adequate capacitance.

Lastly, the radiant power transfer method is the earliest wireless transmission method that has been put to practical use. In this method, the transmitter is absorbed by the radiated receiving antenna and is used by rectifying the resulting power. However, it is the most inefficient method for the reasons such as spreading of the explosive device in every direction and safety restrictions.

The suitability of different power transfer methods for biomedical implants can be discussed according to the re- quirements of the application. Most biomedical implants require power transfer across skin, fat, and a few tissues not exceeding 30-40 mm, Fig. 1.

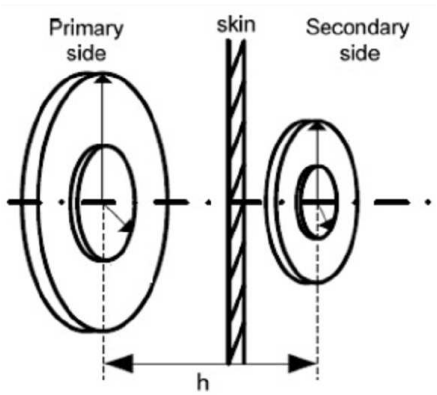

Fig. 1. Transcutaneous power transfer using coils.

IPT, a near field power transfer method for small gaps, is a better choice when it is compared to other methods like radiated power transfer (RPT), which is a far field power transfer method. Small size application, higher power transfer with lower operating frequency (according to $\mathrm{GHz}$ range), meet better the requirements of a typical biomedical implant device. An inductive link contains two electronic components. Primary side includes external battery, power transmitter and control units, secondary units are deep underneath and includes amplifier, rectifier, regulators and power management unit. Power transmission efficiency and system miniaturization are the basic design specifications of a power link. The power transfer efficiency and the magnitude of the power transferred to the load are improved using resonant tuning and matching techniques at the primary and secondary side, respectively. There are four different compensations based on resonance type used in secondary coil. At resonance, the phase of the inductor and capacitor voltages are cancelled. Series-resonant method requires lower voltage swings at its input. Larger voltage oscillation is advantageous for rectifier circuit. The shunt resonant method provides a larger voltage oscillation while series resonant method ensures more current than voltage and accordingly enforces more limitations on the rectifier circuitry which shows the predominant use of shunt resonant method.

\section{Results}

Certain basic parameters such as coupling coefficient, self-inductances and mutual inductance are investigated under magnetostatic conditions, where currents are steady. Both primary and secondary coils are supplied with $50 \mathrm{~mA}$ current. While primary number of turn is 10 , secondary number of turn is 7 , which is shown 
in Fig. 2.

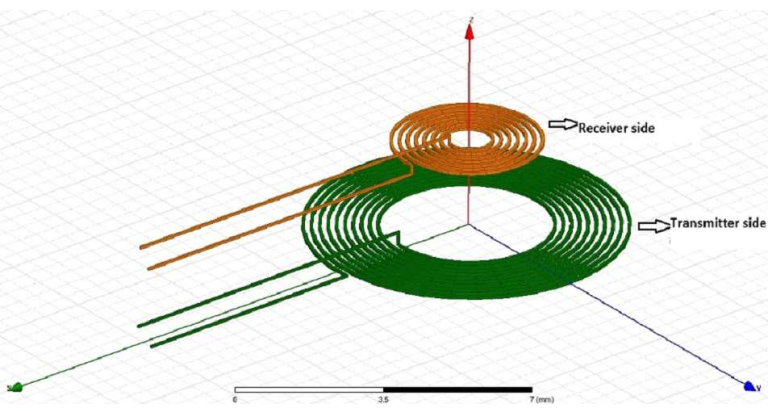

TABLE II

Inductive power link design constraints of a retinal implant system.

\begin{tabular}{|c|c|}
\hline required receive power & $50 \mathrm{~mW}$ \\
\hline $\begin{array}{l}\text { maximum size } \\
\text { (receiver inside the eye) }\end{array}$ & $\begin{array}{c}24 \mathrm{~mm} \\
\text { (eye diameter) }\end{array}$ \\
\hline $\begin{array}{l}\text { operational range } \\
\text { (transmitter-receiver) }\end{array}$ & $20-50 \mathrm{~mm}$ \\
\hline eye movement tolerance & up to $45^{\circ}$ \\
\hline allowable temperature rise & $1^{\circ} \mathrm{C}$ \\
\hline
\end{tabular}

Fig. 2. Concentrically placed coils.

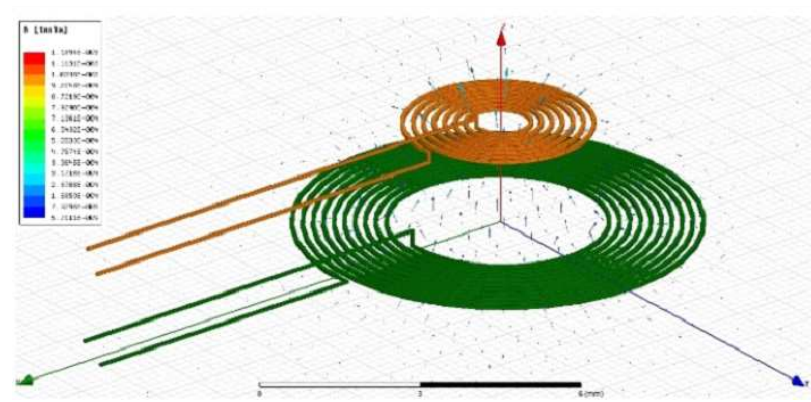

Fig. 3. Magnetic field vector and magnetic field density.

With the spacing of $20 \mathrm{~mm}$, parameters are determined as follows, coupling coefficient: $0.12, \mathrm{~L} 1: 764.78 \mathrm{nH}, \mathrm{L} 2$ : $125.18 \mathrm{nH}, \mathrm{M}: 37.41 \mathrm{nH}$. Magnetic field vector and density are presented in Fig. 3.

It is seen that magnetic field vectors and density are focused on the area between the coils. Retinal implants have a strict size restriction to minimize invasiveness when implanted inside the eye. Implanted transceivers operating in live tissue causes heat increase because of electromagnetic and thermal energy. Electric and magnetic fields are indirectly transformed into heat. Safety limit of them, SAR, is defined by IEEE standard which covers $3 \mathrm{kHz}$ to $3 \mathrm{GHz}$. On the other hand, biocompatibility should be provided in such a way that chemically inert materials are used. The requirements of a typical retinal implant system are briefly stated for wireless power transfer system, Table II.

\section{Conclusion}

Design requires abundant research reviews, mathematical simulations, and benchtop testing with a phantom of the human body, animal studies, and real implants. During the developmental stage, scientific, biological, and medical impasses will be met. There are many factors that affect the efficiency of the system. Among the major factors are impedance and inductance of the coils, relative orientation of the coils, and electromagnetic property of the media surrounding the coils. Using devices with lower frequencies are preferable in terms of temperature rise and SAR limits. To increase the efficiency higher mutual inductances are needed, so intermediate coils could be considered.

\section{References}

[1] M.E. Celik, I. Karagoz, Acta Phys. Pol. A 128, B-297 (2015).

[2] J.C. Schuder, H.E. Stephenson, Jr., IEEE Trans. Biomed. Eng. BME-12, 154 (1965).

[3] J.W. Fuller, IEEE Trans. Biomed. Eng. BME-1, 63 (1968).

[4] C.F. Andren, M.A. Fadali, V.L. Gott, S.R. Topaz, IEEE Trans. Biomed. Eng. BME-15, 278 (1968).

[5] Y. Ahmed, T. Hadjersi, R. Chaoui, Acta Phys. Pol. A 130, 385 (2016).

[6] J. Hirai, T.W. Kim, A. Kawamura, IEEE Trans. Industr. Electron. 46, 349 (1999).

[7] D. Karayel, V. Yegin, Acta Phys. Pol. A 130, 272 (2016).

[8] A. Esser, H.C. Skudelny, IEEE Trans. Industr. Appl. 27, 872 (1991). 